\title{
Perfil del profesional de la información: construcción colaborativa
}

\begin{abstract}
Silvana Grazia Temesio Vizoso
http://orcid.org/0000-0002-3932-8615
\end{abstract}

Diana Comesaña ${ }^{I I}$

http://orcid.org/0000-0002-2623-7343

\section{Graciela Nieto ${ }^{I I I}$}

I Universidad de la República, Uruguay.

Docente Adjunta de Base Datos. Docente Adjunta Redes y sistemas.

II Universidad de la República, Uruguay.

Docente de Redes y Sistemas de Información de las carreras Licenciatura en Bibliotecología y Archivología.

III Universidad de la República, Uruguay.

Docente de Base de datos de las carreras Licenciatura en Bibliotecología y

Archivología.

\section{http://dx.doi.org/10.1590/1981-5344/3693}

El trabajo plantea una metodología para el establecimiento de competencias en un perfil del profesional de la información con la perspectiva de una visión regional que favorezca la movilidad y propenda a un mejoramiento y adecuación a los contextos cambiantes. La metodología toma como marco conceptual la taxonomía de Bloom y establece elementos a completar: áreas de competencias, competencias, categorías temáticas, metodologías enseñanza aprendizaje, evaluación y mapeo de cursos a competencias. Se aplica la propuesta en el área de Documentación Digital del Departamento Tratamiento y Transferencia de Información de la Facultad de 
Información y Comunicaciónen Uruguay. La metodología se plantea como una oportunidad de trabajo colaborativo en la región que se somete a discusión.

Palabras clave: formación bibliotecología; formación archivología; competências; perfil formación; profesional de lainformación.

\section{Information professional profile: collaborative construction}

This paper proposes a methodology to establish competencies in a profile of the information professional with the perspective of a regional vision that favors mobility and tends to improve and adapt to changing contexts. The methodology takes Bloom's taxonomy as a conceptual framework and establishes elements to be completed: areas of competences, competences, thematic categories, teaching-learning methodologies, evaluation and mapping of courses to competences. The proposal is applied in the Digital Documentation area of the Information Processing and Transfer Department of the Faculty of Information and Communication in Uruguay. The methodology is proposed as a collaborative opportunity in the region for discussion.

Keywords: librarianship training; archivist training; profile training; competencies; information training.

\section{Perfil do profissional da informação: construção colaborativa}

O trabalho propõe uma metodologia para estabelecer competências em um perfil do profissional da informação com a perspectiva de uma visão regional que favoreça a mobilidade e tenda a melhorar e se adaptar a contextos 
mutáveis. A metodologia toma a taxonomia de Bloom como um quadro conceitual e estabelece elementos a serem preenchidos: áreas de competências, competências, categorias temáticas, metodologias de ensino-aprendizagem, avaliação e mapeamento de cursos para competências. A proposta é aplicada na área de Documentação Digital do Departamento de Processamento e Transferência de Informações da Faculdade de Informação e Comunicação em Uruguai. A metodologia é proposta como uma oportunidade colaborativa para discussão na região.

Palavras-chave: formação em biblioteconomía; formação em arquivos; competencias; perfil de formação; profissional da informação.

Recebido em 08.10.2018 Aceito em 18.08.2020

\section{Introducción}

La ciencia de lainformación es una disciplina que abreva de otras como matemática, sociologíao estadística. Establecer que comprende cada especialidad y los núcleos de los programas de grado y posgrado, aporta a laclarificación para todos losactores y al relacionamiento a nivel de Latino américa en las reválidas, acreditación y obtención de un perfil latinoamericanoconcalidad educativa.

Existe la necesidad de establecer distintos perfiles, especializaciones, posgrados y competencias profesionales que implica nun relacionamiento con el mercado de trabajo y la visibilización de estos perfiles para acceder a desarrollar tareas que se expanden hacia nuevos puestos y oportunidades a profundizar.

Para realizar un estudio comparativo de los programas de grado y esclarecer la terminología usada para nominar los contenidos es necesario establecer la situación propia. Enese sentido se desarrolla un trabajo a nivel del Departamento Tratamiento y transferencia de información (DTTI) de la Facultad de Información y Comunicación (FIC) en el Instituto de Información (II) de la Universidad de la República (UDELAR) que busca operar de disparador de procesos similares.

Se presentanlos avances realizados dentro del DTTI, relacionado a los aspectos tecnológicos en el ámbito de la Documentación Digital (DD). El planteo no pretende ser un caso cerrado; se ofrece con el mismo criterio de los documentos de Internet Request for Comment (RFC), una 
requisitoria de comentarios para perfilar, con la participación de los actores involucrados, un estado de situación y una propuesta a seguir en forma colaborativa.

Ennuestro Instituto se dictandos licenciaturas: Bibliotecología y Archivología. Queda pendiente la discusión de si es deseable que haya una única carreracon dos especializaciones o dos carreras diferenciadas. Simplemente se señala la situación para establecerel alcance que permea en los desarrollos aquí expuestos. Quedan por fueralos elementos correspondientes a otras disciplinas relacionadas, como museología, archivos médicos u otros que, enlos casos que se entienda de interés, se señalarán.

La propuesta es avanzar en la definición de las competencias que tiene el perfil del profesional de la información (bibliotecólogo y archivólogo) y definir los contenidos que se estudian en la carrera en el área tecnológica.

La ciencia de la información tiene un vasto desarrollo y ha ido creciendo en muchas áreas colaterales que se han ido incorporando, conformando especialidades. Esto supone un desafío para especificar tanto un perfil básico, como perfiles específicos. Cada Institución educativa en América Latina ha desarrollado un proceso diverso por lo que es complejo articular los procedimientos de reválidas entre instituciones y mapear los perfiles profesionales de los egresados o sus especialidades.

Se realizará undetalle de las competencias a partir de las unidades curriculares, sus temas y lacalificación de estas unidades encuanto a la necesidad de formar un perfil básico y/o las distintas especialidades. El alcance es el Departamento Tratamiento y transferencia de Información (DTTI) y específicamente el segmento Documentación Digital (DD) que corresponde a un enfoque tecnológico disciplinar. Se plantea una jerarquía de conocimientos y competencias, para lo que es necesario clarificar los contenidos de todas las unidades curriculares, consensuar una terminología para los temas abordados, definir jerarquías, vínculos, especificidades y términos que puedan ser alternativos, entre otros aspectos.

Con la evolución del conocimiento y el cambio en el rol del profesional, su inserción laboral irá cambiando, por lo que latarea constituye una foto de la situación en un eje temporal. El producto esperado tiene entonces una caracterización cronológica. La propuesta es abordar una fase inicial de un desarrollo futuro que incluya a otras áreas y a otros departamentos del Instituto de Información, otros países e Instituciones y con el lo avanzar hacia un perfil latino americano o una guía para otros procesos como la certificación, la reválida y en general la movilidad como un valor para trascenderlas miradas locales. 


\section{Antecedentes}

El plan de estudios de las licenciaturas de bibliotecología y archivología de 2012 se plantea como una construcción desde distintos ámbitos de cogobierno:

a partir delanálisis de los fundamentos y naturaleza de las disciplinas y lasprofesiones, losefectos de los avances tecnológicos enlasmismas, las características del mercado de trabajoactual y emergente, lasnecesidades y requerimientos de lasociedad $y$ latransformacióndel contexto universitarioactual, de sus políticas y definiciones. (Universidad de la República, 2012)

Con esta perspectiva se estudian los contenidos de las unidades curriculares en conjunto con las metodologías de enseñanza, analizando las competencias y habilidades que de allí se derivan y como éstas son pasibles de ser incorporadas a los ámbitos laborales actuales y proyectados.

Las bibliotecas y archivos como organizaciones acompasan los cambios tecnológicos y sociales pero surgen otras organizaciones 0 emprendimientos donde el profesional de lainformación es un elemento requerido, ya sea solo o formando parte de equipos interdisciplinarios.

El entorno sociocultural y laboral incide en la reformulación de los contenidos para elaborar programas que desarrollen competencias ajustadas a las demandas del mercado de trabajo y al contexto social por lo que hayun ciclo de continua influencia como se detalla en la figura 1.

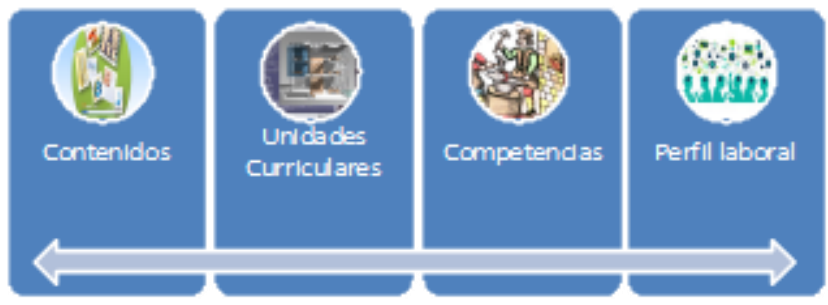

Figura 1. Ciclo de formación e inserción socio-laboral. (Elaboraciónpropia)

El referido plan 2012 establece, a nivel de grado, laformaciónenun núcleo básico disciplinario y busca desarrollar competencias de investigación, especialización y formación continua para que lapráctica académica y profesional se adecue a los cambios tecnológicos y sociales donde este profesional se insertará. Se visualiza un profesional integrado socialmente, que promueve la extensión y el compromiso con las situaciones sociales en las que el profesional adopte un papel activoenlaintegración y transformación social. 
Plantea diversos ejes y define el disciplinar como el núcleo de saberes teórico-prácticos que hacen a laformacióndel título. Los contenidosdel DTTI se correspondenmayoritariamentecon este eje disciplinar.

No existe undesarrolloactual que explicite que contenidos y competencias se dan a nivel de grado o posgrado a nivel regional eneleje tecnológico aunquehay importantes antecedentes que fueron tomados encuenta.

El documento coordinado por la UNAM (Escalona Ríos, 2010) es significativo en este tema. En particular se revisaronlascompetencias (PirelaMorillo, 2010) resultantes del IV Encuentro de Directores y III de Docentes de Escuelas de Bibliotecología y Ciencia de laInformacióndel MERCOSUR, realizado en Montevideo, Uruguay, enelaño 2000. Lascompetenciasenel área de tecnologíatienenlavisión instrumental imperante enesa época que actualmente resulta obsoleta.

Ríos Ortega (2010) señalalanecesidad de:

1.Establecer un sistema legible y comparable de titulaciones.

2.Delimitar conprecisiónlos ciclos de pregrado y postgrado.

3.Diseñar un sistema de créditos.

4.Cooperar enlaregiónlatinoamericana para asegurarestándares de calidadencuanto al desarrollo de criterios y metodologíascomparables.

5.Promover una dimensiónlatinoamericanaenlaeducación superior conénfasiseneldesarrollo curricular.

6.Destacar que un perfil no apunta a homogeneizar los sistemas de educación superior sino que se trata fundamentalmente de incrementar lacompatibilidad de tales sistemas respetandosudiversidad.

Existenalgunosestudios a mencionar como el de Colombia (Zapata Cárdenas, 2007) y más recientemente (Vallejo Sierra, 2016) que analiza pormenorizadamente lasituaciónenColombia estudialoscontenidostroncales de los títulos de grado enInformación y Documentación. A niveleuropeo, el informe ANECA (2004), es undetalladoestudiodel perfil profesional, sus competencias e inserción laboral realizado con una metodología participativa entre la academia y el sector profesional. Lascompetencias que se señalanenel informe para el grado pueden verse enel Anexo 1.

Más recientemente, Borrego (2015) analizalos programas europeos relacionados conBiblioteconomía y Documentación pero sinprofundizarenlos aspectos que nos ocupanen este artículo.

Se han revisado también iniciativas enotrosdominios como el tecnológico que han sido consideradas enestapropuesta (Hawthorne, 2014). 


\section{La tecnología en las carreras delprofesional de la información}

Las TIC en Bibliotecología y Archivología en Uruguay y a nivel del Mercosur tuvieron una etapa en que fueron vistas como elementos transversales e instrumental esa dosadas al concepto de automatización de servicios.

A partir de 2007 la concepción cambió completamente y los programas de estudio de las unidades curriculares Base de datos y Redes y Sistemas plantearon una propuesta diferente. Enel 2012 en oportunidad de las Jornadas de Directores y docentes de bibliotecología del Mercosur se presentó esta visión:

Los procesos que tradicionalmente analiza la ciencia de la información que van desde la ingesta, la estandarización hasta la recuperación, a hora se diversifican: estudiar como nuclear, unir, coordinar, hacer inter operables las piezas de información de todo tipo de soporte o contenido, establecer como se articulan las distintas visiones y flujos de datos, para la ciencia de la información se constituye en una cuestión disciplinar. Aparece un nuevo segmento de contenidos a desarrollar. $Y$ esos desarrollos tienen que incluir en forma in el udible la perspectiva crítica y ética. El debate sobre la libertad de información y el uso del conocimiento se apoya en una perspectiva tecnológica desde lacual se extrapola y es un contenido disciplinar fundacional.

El otro aspecto que plantea el trabajo mencionado es la práctica profesional y el perfil del egresado. Desde nuestra concepción se percibe como una oportunidad de profundizar a nivel de posgrados, con un equilibrio entre lo conceptual y lo práctico que logre adecuar al egresado a las exigencias del mercado laboral y a su vez le abra nuevos campos de acción que le permitan usar con creatividad las herramientas conceptual es con las cuales apoya el conocimiento práctico. (Temesio, 2014)

Esta visión fue debatida y compartida por los docentes del área tecnológica en este Encuentro y a partir de ahí, el eje se reabre y consolida.

Actualmente el eje en Uruguay se adscribe a una tendencia emergente: las Humanidades Digitales (HD), incorporando los conceptos desarrollados. Las HD propendenun aporte innovador y crítico en el uso de la tecnología digital para la gestión y creación de conocimiento y el profesional de la información es un actor primordial en éste ámbito.

A partir de esta visión aparecen especialidades esbozadas como trayectos flexibles en el plan de estudios o como propuestas de educación 
permanente o maestría. Algunas áreas actualmente se están desarrollando en el espacio de DD y se señal an brevemente.

\subsection{Información geográfica}

Con la globalización de los mercados, la fortaleza de una organización reside en sus activos intangible sensu manejo de la información, y la información geográfica, con sus cualidades especiales, constituye una herramienta vital para la toma de decisiones que se transformen en el éxito y supervivencia de las organizaciones públicas y privadas.

Las competencias y experiencia de los especialistas en información, les permiten aportar un valor agregado al proceso de toma de decisiones de las empresas, con la debida inserciónen campos específicos de la información. Muchos de estos nichos laborales emergentes han convertido en protagonistas a profesionales de otras disciplinas por lo cualla formaciónen esta área es estratégica.

\subsection{Información audiovisual}

La documentación audiovisual es un área que atraviesa el mundo analógico, lo digital, las redes, las aplicaciones, los servicios y tiende cada vez más a remezdarseen todos los sentidos, a través de distintos medios y objetos heterogéneos en un mosaico donde el sonido y la imagen forman parte de una forma de expresión instaurada en la comunicación cotidiana. En este panorama se hace necesario realizar una propuesta de abordaje que incluya los múltiples elementos que conforman el tratamiento de la documentación audiovisual.

Uno de los aspectos medulares para eltratamiento de la información audiovisual son sus metadatos. Los metadatos son parte de la cimentación de la arquitectura de la web semántica y constituyen el engranaje para cualquier operación de gestión, búsqueda o difusión.

\subsection{Información semántica}

La web semántica aspira a la recuperación automática de datos, a la interoperabilidad entre comunidades y dominios y al razonamiento automático, a horrando tiempo al usuario y devolviéndole resultados de búsquedas acordes a sus necesidades.

La información semántica, contiene meta-información que acompaña a los documentos en forma de metadatos semánticos estandarizados e incluye el desarrollo de modelos conceptuales de representación de los distintos dominios del conocimiento como las ontologías.

\subsection{Información en Inclusión educativa y pedagogía digital}


La educación, la información y la tecnología contienen elementos que vinculan y se remezclanen una urdimbre intrincada. En este contexto la ciencia de la información es fundamental en aspectos tales como I especificación de metadatos en los recursos educativos y la interoperabilidad informacional en este ecosistema de recursos educativos, entornos virtuales educativos y repositorios. Los aspectos de accesibilidad de los contenidos y los procesos de adecuación forman parte de una postura ética y responsable. Los aspectos técnicos informacionales facilitan la educación inclusiva.

\section{Departamento tratamiento y transferencia de información (DTTI)}

A partir del 13 de agosto de 2015, este Departamento

toma a su cargo la enseñanza, la investigación, la producción de conocimiento y la extensión en lo relativo a: teorías, metodologías, aplicaciones y herramientas para la organización, el procesamiento técnico, el análisis y la recuperación de información en cualquier a de sus formatos y soportes; el dominio y la aplicación de las tecnologías de la información y la comunicación al diseño, creación y administración de productos, servicios y sistemas de información; los principios, métodos de elaboración, revisión y evaluación de repertorios, sistemas, estándares y herramientas de apoyo a la descripción, catalogación, clasificación, indización y condensación; el tratamiento técnico de documentos y recursos de información en cualquier soporte; cuestiones vinculadas al gobierno electrónico, y a redes y sistemas de información. Considera aspectos disciplinarios, multidisciplinarios e interdisciplinarios que enriquecen y complejizan la formación, la investigación y la extensión en el área. (Exp. No 251001-001392-15, 13 ago. 2015).

\subsection{Documentación digital y aplicación de las TIC}

De acuerdo al plan de estudios el módulo Documentación digital y aplicación de las TIC (DD) tiene como objetivos generales:

Brindar fundamentos conceptuales, teorías, especificaciones formales, metodologías y herramientas para la aplicación crítica y ética de las TIC en todas las instancias en que la información y el conocimiento se plasmen en cualquier tipo de soporte y en todo su ciclo de vida. Abordar los aspectos instrumentales de las TIC con una mirada contextualizadora, creativa y desde un punto de vista epistemológico (Universidad de la República, 2012) 
El área de Documentación Digital es el área que involucra todos los elementos tecnológicos que rozan nuestras carreras con un sentido que trasciend el outilitario e incorpora una miradas desde lo conceptual, desde las aproximación teórica y ética constituyendo un área disciplinar.

Esta área vareformulándose con gran flexibilidad en la medida que el contexto y los enfoques enriquecen nuestra disciplina. En este sentido puede verse como una trayectoria hacia las emergentes Humanidades digitales.

Enla tabla 1 se describenlos cursos del área.

\begin{tabular}{|l|l|l|l|l|l|l|l|l|l|l|}
\hline Curso & $\begin{array}{l}\text { Base de } \\
\text { datos }\end{array}$ & $\begin{array}{l}\text { Redes y } \\
\text { sistemas }\end{array}$ & XML & $\begin{array}{l}\text { Intro- } \\
\text { ducción } \\
\text { al } \\
\text { diseño } \\
\text { de } \\
\text { ontolo- } \\
\text { gías }\end{array}$ & $\begin{array}{l}\text { Materiales } \\
\text { Accesi- } \\
\text { bles }\end{array}$ & $\begin{array}{l}\text { Datos } \\
\text { geográ- } \\
\text { ficos }\end{array}$ & ICAATOM & $\begin{array}{l}\text { Herra- } \\
\text { mientas } \\
\text { de } \\
\text { informa- } \\
\text { ción }\end{array}$ & $\begin{array}{l}\text { Funda- } \\
\text { mentos } \\
\text { de la } \\
\text { web } \\
\text { semán- } \\
\text { tica }\end{array}$ & $\begin{array}{l}\text { Gobierno } \\
\text { Eectrónico }\end{array}$ \\
\hline Caracter & $\begin{array}{l}\text { Grado } \\
\text { Obligatorio }\end{array}$ & $\begin{array}{l}\text { Grado } \\
\text { Obligatorio }\end{array}$ & $\begin{array}{l}\text { Grado } \\
\text { optativa }\end{array}$ & $\begin{array}{l}\text { Grado } \\
\text { optativa }\end{array}$ & $\begin{array}{l}\text { Grado } \\
\text { optativa }\end{array}$ & $\begin{array}{l}\text { Grado } \\
\text { optativa }\end{array}$ & $\begin{array}{l}\text { Educa- } \\
\text { ciónperma } \\
\text { n- nente }\end{array}$ & $\begin{array}{l}\text { Educa- } \\
\text { ciónper } \\
\text { man- } \\
\text { nente }\end{array}$ & Maestría & Maestría \\
\hline
\end{tabular}

Tabla 1. Cursos dictados por el área DD

\section{Marco teórico}

Los objetivos de aprendizaje establecen las competencias que se espera obtener a través de las instancias educativas enrelación a los temas o conceptos impartidos. Para la estructuración de los objetivos de aprendizaje se ha utilizado la Taxonomía de Bloom (Bloom, 1971).

El uso de lataxonomía se hace con el fin de que la enunciación del objetivo de aprendizaje sea claro, factible y evaluable. Una vez definidos los objetivos, se planifica la metodología y luego la evaluación.

La estructura de lataxonomía de Bloom es:

-Verbo

-Objeto

En el verbo se tras unta el objetivo y en el objeto se delimita el asunto, que refiere o es lindante a la enunciación de los contenidos. 
La clasificación de Bloom fue revisada por Anderson y Krathwohl dividiendo los objetivos educativos entres dominios: cognitivo (conocimiento), afectivo (actitud) y psicomotor (habilidades) según se documenta enla figura 2.

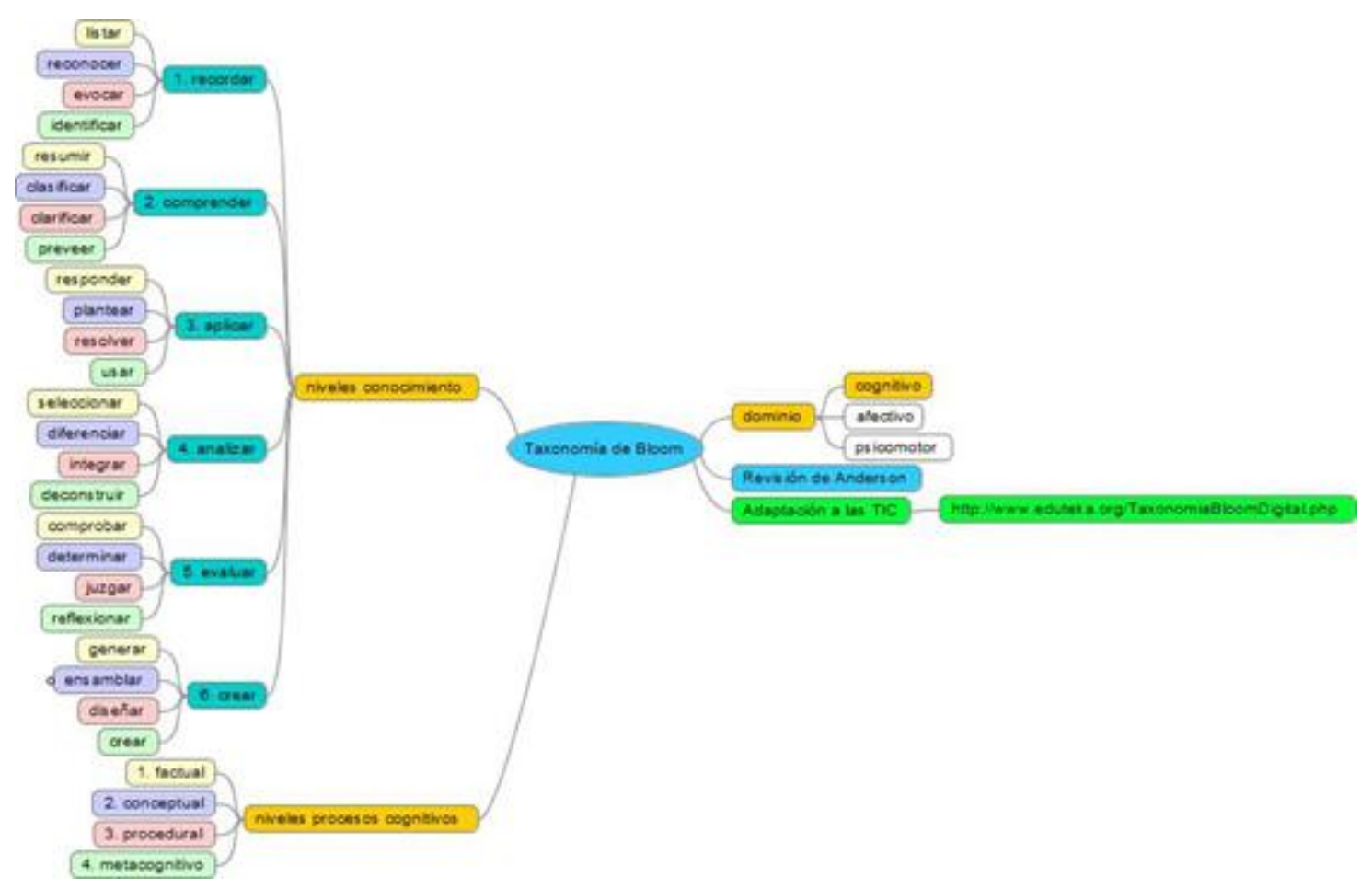

Figura 2. Mapa mental taxonomía de Bloom (Elaboración propia)

El objetivo de aprendizaje toma encuenta el estudiante, sus características, sus necesidades, y sus expectativas de modo de que los objetivos sean factibles, adecuados, y estén alineados con las necesidades relevadas. Asi mismo debe tomarse en cuenta el contexto donde se impartirán porque eso acotará o delimitará los niveles de profundización que se planteen de acuerdo a lataxonomía.

Un aspecto que es importante para la formulación de estos objetivos es que los mismos sean equiparables entre distintos cursos y distintas instituciones, porque el hecho de ser especificados de acuerdo a un estándar, permite establecer niveles de interoperabilidad intra e inter institucional con el beneficio de que el estudiante puede realizar trayectos formativos flexibles dentro de su propia institución o en otra. Los aspectos de flexibilidad y de certificación son importantes del punto de vista formativo y desde el punto de vista formal.

Algunos cursos son presenciales, virtual eso híbridos por lo que surge la cuestión si debe existir alguna diferencia en la enunciación a 
partir de la modalidad. Se entiende que no hay diferencia en la explicitación de los objetivos de aprendizaje entre un curso virtual y uno no virtual, porque la diferencia no está en los objetivos sino en las metodologías y actividades que se desarrollan para cumplirlos. Estas metodologías y actividades son diferentes entre el ámbito presencial y el virtual, pero para cada una de ellas a nivel presencial tienen su correspondiente a nivel virtual, de modo que no resultaría en la diferenciación de un objetivo. El objetivo es una meta de apropiación de conocimiento que se establece para que el estudiante obtenga esa competencia o esa habilidad cognitiva y eso es independiente de las estrategias formuladas para que se produzca esa apropiación.

El estudiante a partir de su trayecto educativo adquiere nuevas habilidades y conocimientos, que constituyen competencias que operan socialmente insertándose en las prácticas laborales.

\section{Metodología}

La metodología propuesta tiene diferentes elementos que se vinculan y se retroalimentan, no necesariamente tienen un desarrollo secuencial. Los elementos son:

- Categorías temáticas

- Competencias en esas categorías

-Contenidos normalizados

-Evaluación (rúbricas)

-Metodologías de enseñanza

-Competenciasen perfiles profesionales

Se busca generar el mapeo de los cursos a las competencias. En lo que sigue se va a bosquejar el desarrollo propuesto e instanciarlo en el área Documentación Digital.

Se busca establecer cuales son las competencias fundamentales o nucleares, tomando encuentalas unidades curriculares del área de DD. De estas unidades se extrajeron las grandes categorías temáticas o agrupación de contenidos medulares y desde allí las competencias que se buscan generar sobre esos contenidos. Estas competencias necesitan ser evaluadas a través de algún patrón que se diseñó como una rúbrica.

Las competencias están ligadas a una categoría temática que agrupa los contenidos y establece una taxonomía como explicitación de granularidad para clarificar los elementos. Las competencias se mapean a cursos y a perfiles laborales. Al terminar el ciclo de todos estos elementos habrá una cohesión y coherencia adecuadas: contenidos en categorías, competencias, evaluación y perfil laboral. Se observó que las 
competencias también están ligadas a las metodologías de enseñanza aprendizaje aunque este elemento no se ha desarrollado en profundidad.

\subsection{Competencias fundamentales del profesional de la informaciónen DD}

Se busca establecer el cuerpo medular del conocimiento en el área denominada documentación digital, que constituye la porción disciplinar del conocimiento de la ciencia de la información que está involucrada y remixada con los aspectos tecnológicos. La idea es delinear conceptos adaptables, no ligados a un enfoque tecnocrático que rápidamente obsolesce.

Se han agrupado en categorías de acuerdo a dominios y luego se detallan en la Tabla 2.

\begin{tabular}{|c|c|}
\hline \multicolumn{2}{|r|}{ 1. Soluciones inform acionales (gobernanza de lainformación) } \\
\hline 1 & Desarrollarestrategias para solucionar problemas informacionales de losusuarios o lasinstituciones \\
\hline 2 & Diferenciar entre las distintas arquitecturas de lainformación y suaplicaciónadecuada \\
\hline 3 & Analizarlosflujos de información a través de distintos procesos y actores \\
\hline 4 & dentificar losestándares de interoperabilidadapropiados para acoplar lostrayectosinformacionales \\
\hline 5 & Identificar las oportunidades de interacción de losactores y lasestrategias de involucramiento \\
\hline 6 & Tmplementar prácticas de seguridad de lainformación y preservación digital. \\
\hline 7 & $\begin{array}{l}\text { Identificar lasestrategiasenel ciclo de vida informacional para modificar, reusar, diversificar y presenvar los recursos } \\
\text { informacionales. }\end{array}$ \\
\hline 8 & $\begin{array}{l}\text { Implementar estrategias para capacitar a losusuariosenlagestión, uso y reutilización de los recursos } \\
\text { informacionales. }\end{array}$ \\
\hline$\overline{9}$ & $\begin{array}{l}\text { Implementar una solución informacional enelescenario a intervenirincluyendolasherramientas, losestándares, los } \\
\text { protocolos , lainteractividad, laseguridad de lainformación y lapreservación digital }\end{array}$ \\
\hline \multicolumn{2}{|c|}{ 2. Sistem as de información } \\
\hline 1 & Definir requerimientos de información para unescenario dado \\
\hline 2 & Definir sistemas de información para el caso relevado \\
\hline$\overline{3}$ & Implementar una solución inf ormacional a medida \\
\hline 4 & Capacitar enel uso de lasolución informacional \\
\hline 5 & Ajustar y mantenerlasolución informacional \\
\hline \multicolumn{2}{|r|}{ 3. Infraestructura de lainform ación } \\
\hline 1 & Tnvestigar laarquitectura de lainformación de los elementos involucrados \\
\hline 2 & Desarrollarelconocimiento de laestructuraabstracta de lainf ormación (MER, UML, ontolog ías, etc.) \\
\hline 3 & Conocerlosmetadatos usados y losmetadatos que se pueden reutilizar y enlazar \\
\hline \multicolumn{2}{|r|}{ 4. Planificación de recursos de inform ación } \\
\hline 1 & Desarrollo de un inventario de información \\
\hline 2 & Desarrollo de un mapa de información \\
\hline 3 & Desarrollo de una propuesta informacional \\
\hline 4 & Desarrollo de unplan de información \\
\hline \multicolumn{2}{|c|}{ 5. Gestióndelconocimiento } \\
\hline 1 & Arquitecturadelconocimiento organizacional \\
\hline 2 & Desarrollo de propuestas de gestióndelconocimiento \\
\hline$\overline{3}$ & Desarrollo de propuestas terminológicas, tesauros y ontologías \\
\hline 4 & Desarrollo de unplan de interoperabilidad informacional \\
\hline \multicolumn{2}{|r|}{ 6. Diseño conceptual de lainformación y suimplantaciónen base de datos } \\
\hline 1 & Describirlasactividades desarrolladasenrelación a lagestión y flujos de datosenunescenario dado \\
\hline$\overline{2}$ & Diagramar undiseño conceptual delescenario relevado. \\
\hline 3 & Comprenderelmapeo de lainformaciónenestructuras de datos y lavinculación entre estos. \\
\hline$\overline{4}$ & Diferenciar los niveles de acceso a losdatos: públicos, privados, perfiles \\
\hline 5 & Implementar eldiseño conceptual enundiseño concreto tomando encuentarestricciones de consistencia \\
\hline 6 & Prototiparescenarios de consulta y suimplementaciónpráctica. \\
\hline 7 & Realizar tareas administrativas de gestión de base de datos \\
\hline
\end{tabular}




\begin{tabular}{|c|c|}
\hline 8 & Realizar eltesteo de larecuperación de lainformación y su ajuste \\
\hline \multicolumn{2}{|c|}{ 7. Redes de información } \\
\hline 1 & Comprenderlacomplejidad de un problema de redes y sus diversas capas. \\
\hline 2 & Describirlas capas, loslímites, los protocolos y las distintas topologías de las redes de información. \\
\hline 3 & Diagramar los componentes y las características de una red informacional. \\
\hline 4 & Resumir elflujo de datos a través de unescenario de red informacional \\
\hline 5 & Analizarlacomunicación y los aspectos de infraestructura y topología \\
\hline 6 & Analizarlainteroperabilidad de lainformación \\
\hline 7 & Analizarlaestructura de lainformación \\
\hline 8 & Analizarlos sistemas de información \\
\hline \multicolumn{2}{|c|}{ 8. Atención al usuario } \\
\hline 1 & Demostrar lasmejoresprácticasenlos sistemas de información o las redes de información. \\
\hline 2 & Demostrar los problemas de seguridad de lainformación y ciudadanía digital \\
\hline 3 & Discutir los aspectos de ética de lainformaciónenlos sistemas informacionales, las redes y lasactividadesdigitales \\
\hline 4 & Discutir los aspectos de seguridad de lainformación y preservación digital y realizar capacitación \\
\hline 5 & Discutir los aspectos de gobernanza de datos \\
\hline \multicolumn{2}{|r|}{ 9. Equipos interdisciplinarios para elcumplimiento de objetivos comunes } \\
\hline 1 & Utilizar lacomunicación, latolerancia, lanegociación, lascompetencias colaborativas y sociales \\
\hline \multicolumn{2}{|r|}{ 10. Competenciassociales y comunicativas } \\
\hline 1 & $\begin{array}{l}\text { Desarrollar habilidades para interpretar lainformación técnica, expresarseconcorrección, establecer una } \\
\text { comunicación oraly escrita adecuada a laaudienciaen particular. }\end{array}$ \\
\hline 2 & $\begin{array}{l}\text { Producirmanuales, formularios y documentación técnica identificando laaudiencia de modo que el material } \\
\text { seainteligible. }\end{array}$ \\
\hline 3 & Utilizar ladocumentación y elconocimiento para resolver desafíosen entornos informacionales. \\
\hline 4 & $\begin{array}{l}\text { Aprender a trabajaren grupo colaborando y respetandoladiversidad y ejerciendolatolerancia y latransparencia } \\
\text { comunicativa }\end{array}$ \\
\hline \multicolumn{2}{|c|}{ 11. Competenciasprofesionales } \\
\hline 1 & Discutir tendencias y desarrollos emergentes y su impacto social con una actitud crítica y socialmente involucrada. \\
\hline 2 & Tnvolucrarseenelaprendzaje continuo y lainvestigación, valorar críticamentelosnuevosescenarios y propuestas. \\
\hline 3 & Habilidadendesarrollar soluciones innovadoras a problemas nuevos o escenariosnuevos. \\
\hline \multicolumn{2}{|c|}{ 12. Desempeñoprofesional y ético } \\
\hline 1 & Comprenderlasimplicancias éticas de lagestión de lainformación y lasrepercusionessociales y políticas de lamisma \\
\hline 2 & Tener una conductaprof esional ética e involucrada socialmente \\
\hline 3 & $\begin{array}{l}\text { Promover losdesarrollosprofesionales y éticos de las sociedades profesionalesnacionales e internacionales. (IFLA e } \\
\text { ICA) }\end{array}$ \\
\hline \multicolumn{2}{|r|}{ 14. Demostrar eficiencia laboral, creatividad y emprendedurismo } \\
\hline 1 & Desarrollarmejoras y propuestas enlos aspectos informacionalesenlosprocesos y losservicios. \\
\hline 2 & $\begin{array}{l}\text { Desarrollarel rol delprof esional de lainformaciónenelcumplimiento de lamisión y los roles de laorganizaciónenla que } \\
\text { se desempeña }\end{array}$ \\
\hline
\end{tabular}

Tabla 2. Competencias nucleares del área DD. Elaboraciónpropia

\subsection{Metodologías de enseñanza}

Las metodologías acompañan el desarrollo y la evaluación y son particularmente importantes en este tipo de materias. Podemos mencionar las metodologías que utilizamos en los cursos de grado:

Bases de datos: Se proponeun tema y se desarrolla como un proyecto con varios hitos en los que reciben retroalimentación. La propuesta teórica y práctica se instancia en una situación real que deben investigar constituyendo una prestamiento a la investigación.

Se incluyen dentro de las estrategias didácticas juegos que se incorporan como desempeños físicos que pueden ser luego evocados.

Redes y sistemas: Tienen cuatro instancias de entrega: 3 entregas con una arquitectura de información dada y con el desarrollo de una instancia práctica y un informe sobre las decisiones y elementos que se 
tomaron de acuerdo al caso. La cuarta entrega es el desarrollo de una solución informacional nueva que investigan y presentan en clase abierta. Se complementa con seminarios internos de discusión de temas de actualidad.

\subsection{Evaluación: Rúbricas}

Las competencias se complementan con la evaluación a través de una rúbrica de acuerdo a 3 niveles: mínimo, adecuado, excelente. Por ejemplo, en la categoría Diseño conceptual de la información y su implantación en base de datos, y, la competencia 3 Comprender el mapeo de la información en estructuras de datos y la vinculación entre estos, la rúbrica sería del estilo siguiente:

Mínimo: tiene el concepto de estructura de datos y vínculos aunque lo aplica con dificultad

Adecuado: Diseña adecuadamente las estructuras de datos

Excelente: Diseñalas estructuras de datos con solvencia y es capaz de visualizar los problemas y las restricciones que se plantean

\subsection{Mapear competencias a cursos}

La propuesta es que una vez acordadas categorías y competencias, se mape en las competencias a los cursos de una entidad educativa y sus respectivas carreras, en este caso la Facultad de Información y Comunicación (FIC) y sus carreras Bibliotecología (B) y Archivología (A) como se muestra parcialmente en la tabla 3.

\begin{tabular}{|l|l|}
\hline Compe tencias & Curs os \\
\hline $\begin{array}{l}\text { 6.1 Describirlasactividadesdesarrolladasenrelación a lagestión } \\
\text { y flujos de datosenunescenario dado }\end{array}$ & Base de datos (FIC: B,A) \\
\hline $\begin{array}{l}\text { 12.2 Tener una conductaprofesional ética e involucrada } \\
\text { socialmente }\end{array}$ & Base de datos (FIC: B,A) \\
\hline $\begin{array}{l}\text { 12.2 Tener una conductaprof esional ética e involucrada } \\
\text { socialmente }\end{array}$ & Redes y sistemas (FIC: B,A) \\
\hline$\ldots$ & \\
\hline
\end{tabular}

Tabla 3. Mapeo de lascompetencias a los cursos en FIC

\subsection{Categorías temáticas}

La tarea futura es realizar una categorización general de los tópicos fundamentales impartidos en las carreras de ciencia de la información en el Mercosur, para lograr la correlación de conceptos y competencias equiparable. Esta categorización general serviría como puesta a punto de los temas que se están impartiendo en las carreras y reflejarí aun estado del arte de los programas, constituyendo una herramienta flexible adecuada a loscambios.

A modo de ejemplo de esta categorización que nos permita establecer un patrón de los contenidos podría esbozarse: SISTEMAS DE 
INFORMACIÓN que se abre en: Sistemas integrados de gestión bibliotecaria, Sistemas de descripción archivística, Gestión de colecciones digitales, Gestores documentales, Gestores de contenidos, Gestores de proyectos, Repositorios documentales, Repositorios de datos, Sistemas web 2.0 que se abre en Blogs, Wikis ..., etc.

Este desarrollo no busca ser una propuesta, sino simplemente une jemplo y línea de trabajo futura con la idea de construir una taxonomía, tesauro u ontología sobre los contenidos de la carrera que homogeinice la variación de los nombres de los temas en los programas en nuestras carreras.

Si se llega a una cuerdo respecto de las competencias que son representadas en los contenidos, es posible generar un estándar que favorezca la movilidad tanto a nivel estudiantil y docente como profesional. Esta situación se trasladaría al entorno laboral y académico con las consiguientes ventajas de proyectos e investigación regional.

\subsection{Perfil laboral}

No puede so slayarseel entorno laboral en que el profesional que se forma debe insertarse y donde las competencias desarrolladas constituirá nun valor. El mercado laboral, el marco social-cultural inciden en la formación impartida. Si bienel planteo educativo va más alládel perfil laboral, y, en particular, el perfil laboral actual, no puede negarse que existe una interacción entre las competencias y las categorías temáticas y que existe una gran influencia. En el caso de lascarreras que comprend en la ciencia de la información el explicitar lo puede ser un elemento beneficioso para el profesional, porque generalmente se asocia al profesional de la información con un mercado de trabajo que condice con las instituciones tradicionales y no se percibe claramente el beneficio de su inclusión en ámbitos no tan tradicionales.

Se muestra un fragmento de la propuesta en la tabla 4.

\begin{tabular}{|l|l|}
\hline Competencias & Perfillaboral \\
\hline $\begin{array}{l}\text { 2.1 Describirlasactividadesdesarrolladasenrelación a } \\
\text { lagestión y flujos de datosenunescenario dado }\end{array}$ & $\begin{array}{l}\text { Institucionescomerciales, culturales y de } \\
\text { gobierno que se planteenlagobernanza de } \\
\text { lainformación para lamejora de sus procesos de } \\
\text { negocio y eldesarrollo de planes estratégicos }\end{array}$ \\
\hline$\ldots$ & \\
\hline
\end{tabular}

Tabla 4. Mapeo de competencias al perfil laboral

\subsection{Desarrollodel caso DD}

En el ANEXO 2 se presentan las competencias del área de DD de las materias troncales.

\section{Conclusiones y trabajos futuros}


Si esta iniciativa se propaga, se podrán establecer criterios comunes que propendan a un mejoramiento de los programas de estudio y a una estandarización de criterios en los aspectos medulares para poder tender a una normalización y a un nivel de calidade quiparable.

Nuestra región marca diferencias de enfoque cultural, social e ideológico con otras partes del mundo. Una iniciativa de este tipo que permita conocernos, compartir nuestras prácticas, intercambiar enfoques y experiencias con una mirada socialmente involucrada con la perspectiva de la calidad técnica permitirá un mejoramiento de la inserción social y laboral de los egresados.

La perspectiva hacia el desempeño profesional y el mercado de trabajo es un enfoque a desarrollar. Este enfoque no debe ser el único porque, por un lado es inadecuado dado que la evolución de la tecnología y la innovación impactan en el mercado de trabajo rápidamente. Una instantánea de lo que hoy se plantea será desajustada u obsoleta en poco tiempo. Por otra parte los objetivos educativos tienen una mirada en otros aspectos que trascienden lo utilitario, es necesaria la formación teórica, la investigación, la mirada socialmente involucrada con los desfavorecidos, los aspectos éticos, ideológicos y políticos, entre otros asuntos que no necesariamente estarán contemplados en una mirada hacia las competencias buscadas en el mercado de trabajo.

Los aspectos tecnológicos en nuestras carreras han sufrido variaciones muy importantes y éstas no han sido recogidas o asimiladas en todo su espectro. La tecnologíaya está incorporada pero con un sentido instrumental, es necesario profundizar en el pensamiento computacional como un elemento que nos ayude a desarrollar el marco teórico disciplinar que no está abordado con profundidad en los programas de estudio.

Una iniciativa como la propuesta favorecería la movilidad tanto de estudiantes como de docentes mejorando la calidad de la formación y la formulación de una ciencia de la información latinoamericana.

Si se produce adhesión a esta iniciativa se puede estructurar un sitio web colaborativo de construcción colectiva que implemente la participación para la formulación de esta propuesta, incluyendo todos los elementos metodológicos planteados.

\section{Referencias}

Agencia Nacional de Evaluación de laCalidad y Acreditación (ANECA). Libro Blanco: Título de grado en información y documentación. 2004.

Disponibleen:

http://www.aneca.es/var/media/150424/libroblanco jun05 documentacio n.pdf. Consultado 05 oct. 2018 
ANDERSON. W. L., David, R. \&Krathwohl, D. R. Una taxonomía para elaprendizaje, enseñanza y evaluación: una revisión de lataxonomía de Bloom de objetivos educativos. 2001. Nueva York: Longman.

BANCAYÁN ORÉ, C. Operacionalización de lataxonomía de Anderson y Krathwohl para la docencia universitaria. En: Paideia., v. 3, n 4, 2017. p. 109-119.

BLOOM, B. S. Taxonomía de los objetivos de laeducación; la clasificación de las metas educacionales. Manuales I y II. 1971.

BORREGO, À. Elsestudis de Biblioteconomia i Documentació a Europa : una visió general. BiD: textos universitaris de biblioteconomia $\mathrm{i}$ documentació, n. 35 des - 2015. Disponible en: http://bid.ub.edu/es/35/borrego.htm. Consultado 05 oct. 2018

Exp. No 251001-001392 - (Distribuido No 731/15). Facultad de Información y Comunicación. Comisióndel Instituto de Información. 13 de Agosto de 2015. Estructura Académica del Instituto de Información. Disponible en: DOI:

http://www.expe.edu.uy/expe/resoluci.nsf/f7a4bee664a9738b032582570 0465596/e8de11306484fb9f83257e9d005960e1?OpenDocument. Consultado 05 oct. 2018

HAWTHORNE, E. et al Information Technology Competency Model of Core Learning Outcomes and Assessment for Associate-Degree Curriculum. ACM. 2014. Disponible en: http://ccecc.acm.org/files/publications/ACMITCompetencyModel140ctober 201420150114T180322.pdf. Consultado 05 oct. 2018

Las competencias en el perfil bibliotecológico en América Latina. coord. Lina Escalona Ríos. 2010, México : UNAM, Centro Universitario de Investigaciones Bibliotecológicas. viii, 94 p. - (Cuadernos de Investigación ; 14)

MAGER, R. F. Mager's Tips on Instructional Objectives.Preparing instructional objectives. (2nd ed.). 1984. Belmont, CA: David S. Lake. Disponible en:http://www2.gsu.edu/ mstmbs/CrsTools/Magerobj.html. Consultado 05 oct. 2018

PIRELA MORILLO, J. Los perfiles profesionales por competenciasenBibliotecología, Archivología y Ciencia de laInformación. En: Las competencias en el perfil bibliotecológico en América Latina / coord. Lina Escalona Ríos. 2010. México : UNAM, Centro Universitario de Investigaciones Bibliotecológicas. viii, 94 p. (Cuadernos de Investigación ; 14) 
REGILLO, R. Unvistazo al estado actual de la Web Semántica.2018. Disponible en: https://www.beeva.com/beeva-view/innovacion/unvistazo-al-estado-actual-de-la-web-semantica/. Consultado 05 oct. 2018

RÍOS ORTEGA, J. Perfil de competencias en bibliotecología para la región latinoamericana: exordio a sue laboración. En: Las competencias en el perfil bibliotecológico en América Latina / coord. Lina Escalona Ríos. México : UNAM, Centro Universitario de Investigaciones Bibliotecológicas, 2010. viii, 94 p. (Cuadernos de Investigación ; 14). Disponible en: http://libros.metabiblioteca.org/bitstream/001/218/8/competencias perfil .pdf. Consultado 05 oct. 2018

TEMESIO, S,Comesaña, Diana, Nieto, Graciela. Las TIC en la ciencia de la información y en e Inuevo plan de estudios de la EUBCA. EnREBECIN Revista brasileira de educacao en Ciencia da Informacao, v1, n1, 2014. p. 35-47 Disponible en:

http://abecin.org.br/portalderevistas/index.php/rebecin/article/view/6/pdf 1. Consultado 05 oct. 2018

Universidad de la República.Escuela Universitaria de Bibliotecología y Ciencias Afines. Plan de estudios para las carreras de grado de la EUBCA, Licenciatura en Bibliotecología y Licenciatura en Archivología. 2012. Disponible en: http://fic. edu.uy/sites/default/files/inlinefiles/Plan\%20Estudios\%20Lic\%20Bibliotecologia\%20\%20y\%20Lic\%20ArC hivologia.pdf. Consultado 05 oct. 2018

VALLEJO SIERRA, R. H. Hacia una formación común en Bibliotecología: el caso colombiano. En:Bibliotecas: Revista de la Escuela de Bibliotecología, Documentación e Información, [S.I.], v. 34, n. 2, dec. 2016. p. 1-28. Disponible en:

http://www.revistas.una.ac.cr/index.php/bibliotecas/article/view/8953/11 049.Consultado 05 oct. 2018

World Wide Web Consortium. Guía Breve de Web Semántica. 2017. Disponible en:https://www.w3c.es/Divulgacion/GuiasBreves/WebSemantica. Consultado 05 oct. 2018

ZAPATA CÁRDENAS, C. A. La oferta formativa en bibliotecología en Colombia: análisis actualdel sector. En: Revista Interamericana de Bibliotecología, v. 30, n. 2, Jul.- Dic, 2017. p.165-188. Disponible en:http://www.scielo.org.co/pdf/rib/v30n2/v30n2a08.pdf. Consultado 05 oct. 2018 


\section{ANEXO 1 - Competencias de grado de acuerdo al Libro Blanco (ANECA, 2004) referentes al título de Biblioteconomía y Documentación}

\begin{tabular}{|c|c|c|}
\hline \multirow{3}{*}{ 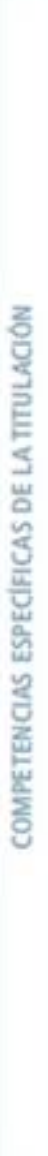 } & & 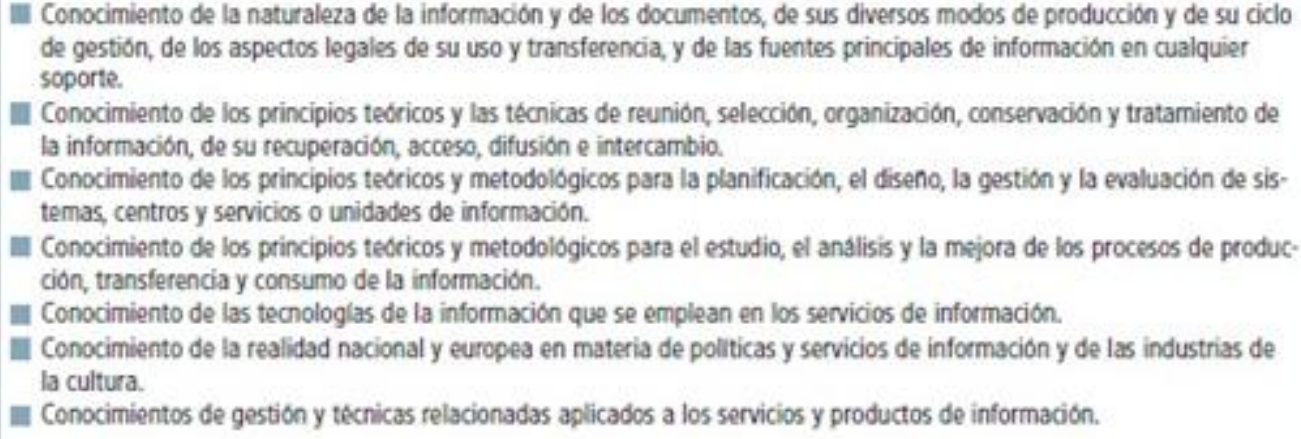 \\
\hline & $\frac{\frac{8}{2}}{\frac{e^{2}}{\frac{8}{2}}}$ & 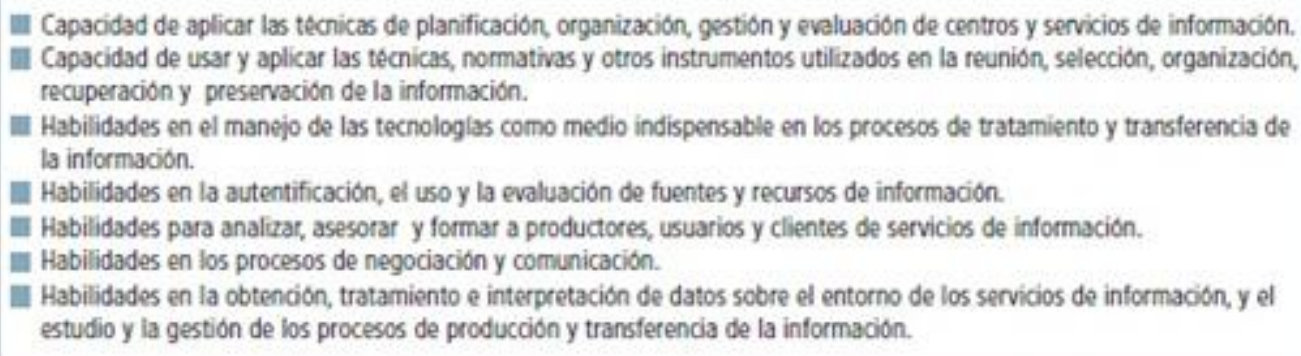 \\
\hline & 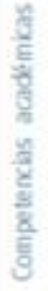 & 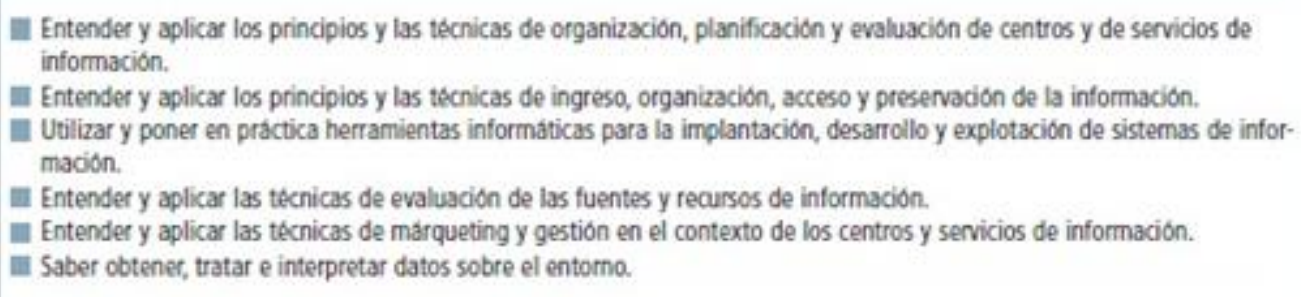 \\
\hline \multirow{3}{*}{ 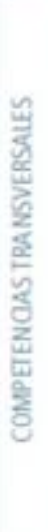 } & 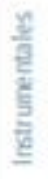 & 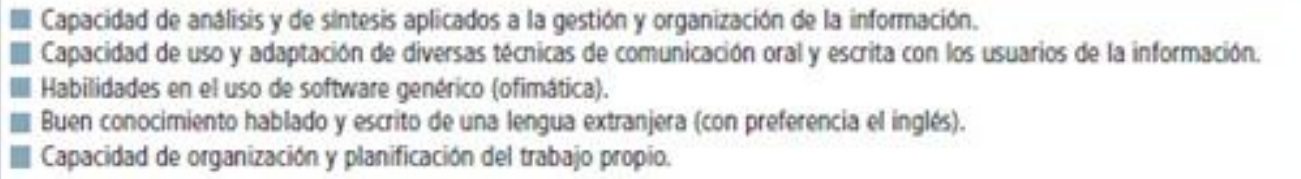 \\
\hline & $\frac{\frac{x}{2}}{\frac{8}{2}}$ & $\begin{array}{l}\text { Capacidad de trabajar en equipo y de integracion en equipos multidisciplinares. } \\
\text { Reconocimiento de la diversidad y la multiculturalidad en el trabajo en el servicio póblico. } \\
\text { a Razonamiento critico en el analisis y la valoración de aiternativas. } \\
\text { in Compromiso etico en I as relaciones con los usuarios y en la gestion de la informacion. }\end{array}$ \\
\hline & 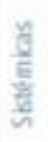 & 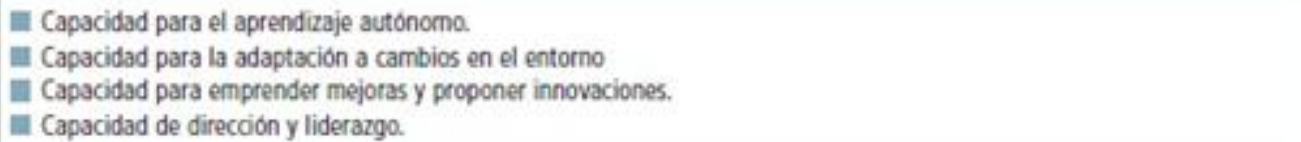 \\
\hline
\end{tabular}




\section{ANEXO 2. Competenciastroncales área Documentación Digital}

\begin{tabular}{|c|c|}
\hline $\begin{array}{l}\text { 1. Soluciones informacionales (gobernanza de } \\
\text { lainformación) }\end{array}$ & Cursos \\
\hline $\begin{array}{l}\text { Desarrollarestrategias para solucionar problemas } \\
\text { informacionales de losusuarios o lasinstituciones }\end{array}$ & $\begin{array}{l}\text { Base de datos } \mathrm{FIC}(\mathrm{B}, \mathrm{A}) \\
\text { Redes y sistemas } \mathrm{FIC}(\mathrm{B}, \mathrm{A})\end{array}$ \\
\hline $\begin{array}{l}\text { Diferenciar entre las distintas arquitecturas de } \\
\text { lainformación y suaplicaciónadecuada }\end{array}$ & Redes y sistemas FIC $(\mathrm{B}, \mathrm{A})$ \\
\hline $\begin{array}{l}\text { Analizarlosflujos de información a través de distintos } \\
\text { procesos y actores }\end{array}$ & Redes y sistemas FIC $(\mathrm{B}, \mathrm{A})$ \\
\hline $\begin{array}{l}\text { Identificar losestándares de interoperabilidadapropiados } \\
\text { para acoplar lostrayectosinformacionales }\end{array}$ & Redes y sistemas FIC $(B, A)$ \\
\hline $\begin{array}{l}\text { Identificar las oportunidades de interacción de losactores y } \\
\text { lasestrategias de involucramiento }\end{array}$ & Redes y sistemas FIC $(B, A)$ \\
\hline $\begin{array}{l}\text { Implementar prácticas de seguridad de lainformación y } \\
\text { preservación digital. }\end{array}$ & Redes y sistemas FIC $(\mathrm{B}, \mathrm{A})$ \\
\hline $\begin{array}{l}\text { Identificar lasestrategiasenel ciclo de vida informacional } \\
\text { para modificar, reusar, diversificar y preservar los recursos } \\
\text { informacionales. }\end{array}$ & Redes y sistemas FIC $(\mathrm{B}, \mathrm{A})$ \\
\hline $\begin{array}{l}\text { Implementar estrategias para capacitar a } \\
\text { losusuariosenlagestión, uso y reutilización de los recursos } \\
\text { informacionales. }\end{array}$ & $\begin{array}{l}\text { Base de datos FIC }(B, A) \\
\text { Redes y sistemas FIC }(B, A)\end{array}$ \\
\hline $\begin{array}{l}\text { Implementar una solución informacional enelescenario a } \\
\text { intervenirincluyendolasherramientas, losestándares, los } \\
\text { protocolos, lainteractividad, laseguridad de lainformación y } \\
\text { lapreservación digital }\end{array}$ & $\begin{array}{l}\text { Base de datos FIC }(B, A) \\
\text { Redes y sistemas } \mathrm{FIC}(B, A)\end{array}$ \\
\hline 2. Sistemas de información & \\
\hline $\begin{array}{l}\text { Definir requerimientos de información para unescenario } \\
\text { dado }\end{array}$ & $\begin{array}{l}\text { Base de datos FIC }(B, A) \\
\text { Redes y sistemas FIC }(B, A)\end{array}$ \\
\hline Definir sistemas de información para el caso relevado & Redes y sistemas FIC $(\mathrm{B}, \mathrm{A})$ \\
\hline Implementar una solución informacional a medida & Redes y sistemas FIC $(\mathrm{B}, \mathrm{A})$ \\
\hline Capacitar enel uso de lasolución informacional & $\begin{array}{l}\text { Base de datos } \mathrm{FIC}(\mathrm{B}, \mathrm{A}) \\
\text { Redes y sistemas } \mathrm{FIC}(\mathrm{B}, \mathrm{A})\end{array}$ \\
\hline Ajustar y mantenerlasolución inf ormacional & $\begin{array}{l}\text { Base de datos FIC }(B, A) \\
\text { Redes y sistemas } \mathrm{FIC}(\mathrm{B}, \mathrm{A})\end{array}$ \\
\hline 3. Infraestructura de lainformación & \\
\hline $\begin{array}{l}\text { Investigar laarquitectura de lainformación de los elementos } \\
\text { involucrados }\end{array}$ & Redes y sistemas FIC $(\mathrm{B}, \mathrm{A})$ \\
\hline $\begin{array}{l}\text { Desarrollarelconocimiento de laestructuraabstracta de } \\
\text { lainformación (MER, UML, ontologías, etc.) }\end{array}$ & $\begin{array}{l}\text { Base de datos FIC }(B, A) \\
\text { Redes y sistemas FIC }(B, A)\end{array}$ \\
\hline $\begin{array}{l}\text { Conocerlosmetadatos usados y losmetadatos que se } \\
\text { pueden reutilizar y enlazar }\end{array}$ & $\begin{array}{l}\text { Base de datos FIC }(B, A) \\
\text { Redes y sistemas } \mathrm{FIC}(\mathrm{B}, \mathrm{A})\end{array}$ \\
\hline 4. Planificación de recursos de información & \\
\hline Desarrollo de un inventario de información & Redes y sistemas FIC $(\mathrm{B}, \mathrm{A})$ \\
\hline Desarrollo de un mapa de información & Redes y sistemas FIC $(\mathrm{B}, \mathrm{A})$ \\
\hline Desarrollo de una propuesta informacional & Redes y sistemas FIC $(B, A)$ \\
\hline Desarrollo de unplan de información & Redes y sistemas FIC $(\mathrm{B}, \mathrm{A})$ \\
\hline
\end{tabular}




\begin{tabular}{|c|c|}
\hline 5. Gestióndelconocimiento & \\
\hline Arquitecturadelconocimiento organizacional & Redes y sistemas FIC $(\mathrm{B}, \mathrm{A})$ \\
\hline Desarrollo de propuestas de gestióndelconocimiento & Redes y sistemas FIC $(\mathrm{B}, \mathrm{A})$ \\
\hline $\begin{array}{l}\text { Desarrollo de propuestas terminológicas, tesauros y } \\
\text { ontologías }\end{array}$ & Redes y sistemas FIC $(B, A)$ \\
\hline Desarrollo de unplan de interoperabilidad informacional & Redes y sistemas FIC $(\mathrm{B}, \mathrm{A})$ \\
\hline $\begin{array}{l}\text { 6. Diseño conce ptual de lainformación y } \\
\text { suimplantaciónen base de datos }\end{array}$ & \\
\hline $\begin{array}{l}\text { Describirlasactividades desarrolladasenrelación a lagestión } \\
\text { y flujos de datosenunescenario dado }\end{array}$ & Base de datos FIC $(B, A)$ \\
\hline Diagramar undiseño conceptual delescenario relevado. & Base de datos FIC $(B, A)$ \\
\hline $\begin{array}{l}\text { Comprenderelmapeo de lainformaciónenestructuras de } \\
\text { datos y lavinculación entre estos. }\end{array}$ & Base de datos FIC $(B, A)$ \\
\hline $\begin{array}{l}\text { Diferenciar los niveles de acceso a losdatos: públicos, } \\
\text { privados, perfiles }\end{array}$ & Base de datos FIC $(B, A)$ \\
\hline $\begin{array}{l}\text { Implementar eldiseño conceptual enundiseño concreto } \\
\text { tomando encuentarestricciones de consistencia }\end{array}$ & Base de datos FIC $(B, A)$ \\
\hline $\begin{array}{l}\text { Prototiparescenarios de consulta y } \\
\text { suimplementaciónpráctica. }\end{array}$ & Base de datos FIC $(B, A)$ \\
\hline $\begin{array}{l}\text { Realizar tareas administrativas de gestión de base de } \\
\text { datos }\end{array}$ & Base de datos FIC $(B, A)$ \\
\hline $\begin{array}{l}\text { Realizar eltesteo de larecuperación de lainformación y su } \\
\text { ajuste }\end{array}$ & Base de datos FlC $(B, A)$ \\
\hline 7. Redes de información & \\
\hline $\begin{array}{l}\text { Comprenderlacomplejidad de un problema de redes y sus } \\
\text { diversas capas. }\end{array}$ & Redes y sistemas FIC $(\mathrm{B}, \mathrm{A})$ \\
\hline $\begin{array}{l}\text { Describirlas capas, loslímites, los protocolos y las distintas } \\
\text { topologías de las redes de información. }\end{array}$ & Redes y sistemas FIC $(\mathrm{B}, \mathrm{A})$ \\
\hline $\begin{array}{l}\text { Diagramar los componentes y las características de una } \\
\text { red informacional. }\end{array}$ & Redes y sistemas FIC $(B, A)$ \\
\hline $\begin{array}{l}\text { Resumir elflujo de datos a través de unescenario de red } \\
\text { informacional }\end{array}$ & Redes y sistemas FIC $(\mathrm{B}, \mathrm{A})$ \\
\hline $\begin{array}{l}\text { Analizarlacomunicación y los aspectos de infraestructura y } \\
\text { topología }\end{array}$ & Redes y sistemas FIC $(B, A)$ \\
\hline Analizarlainteroperabilidad de lainformación & Redes y sistemas FIC $(\mathrm{B}, \mathrm{A})$ \\
\hline Analizarlaestructura de lainformación & Redes y sistemas FIC $(\mathrm{B}, \mathrm{A})$ \\
\hline Analizarlos sistemas de información & Redes y sistemas FIC $(\mathrm{B}, \mathrm{A})$ \\
\hline 8. Atención al usuario & \\
\hline $\begin{array}{l}\text { Demostrar lasmejoresprácticasenlos sistemas de } \\
\text { información o las redes de información. }\end{array}$ & $\begin{array}{l}\text { Base de datos FIC }(B, A) \\
\text { Redes y sistemas FIC }(B, A)\end{array}$ \\
\hline $\begin{array}{l}\text { Demostrar los problemas de seguridad de lainformación y } \\
\text { ciudadanía digital }\end{array}$ & $\begin{array}{l}\text { Base de datos FIC }(B, A) \\
\text { Redes y sistemas } \mathrm{FIC}(B, A)\end{array}$ \\
\hline $\begin{array}{l}\text { Discutir los aspectos de ética de lainformaciónenlos } \\
\text { sistemas informacionales, las redes y } \\
\text { lasactividadesdigitales }\end{array}$ & $\begin{array}{l}\text { Base de datos FIC }(B, A) \\
\text { Redes y sistemas FIC }(B, A)\end{array}$ \\
\hline $\begin{array}{l}\text { Discutir los aspectos de seguridad de lainformación y } \\
\text { preservación digital y realizar capacitación }\end{array}$ & $\begin{array}{l}\text { Base de datos FIC }(B, A) \\
\text { Redes y sistemas } F I C(B, A)\end{array}$ \\
\hline Discutir los aspectos de gobernanza de datos & $\begin{array}{l}\text { Base de datos FIC }(B, A) \\
\text { Redes y sistemas FIC }(B, A)\end{array}$ \\
\hline
\end{tabular}




\begin{tabular}{|c|c|}
\hline $\begin{array}{l}\text { 9. Equipos interdisciplinarios para elcumplimiento } \\
\text { de objetivos comunes }\end{array}$ & \\
\hline $\begin{array}{l}\text { Utilizar lacomunicación, latolerancia, lanegociación, } \\
\text { lascompetencias colaborativas y sociales }\end{array}$ & $\begin{array}{l}\text { Base de datos FIC }(B, A) \\
\text { Redes y sistemas FIC }(B, A)\end{array}$ \\
\hline 10. Competenciassociales y comunicativas & \\
\hline $\begin{array}{l}\text { Desarrollar habilidades para interpretar lainformación } \\
\text { técnica, expresarseconcorrección, establecer una } \\
\text { comunicación oraly escrita adecuada a laaudienciaen } \\
\text { particular. }\end{array}$ & $\begin{array}{l}\text { Base de datos FIC }(B, A) \\
\text { Redes y sistemas FIC }(B, A)\end{array}$ \\
\hline $\begin{array}{l}\text { Producirmanuales, formularios y documentación técnica } \\
\text { identificando laaudiencia de modo que el material } \\
\text { seainteligible. }\end{array}$ & $\begin{array}{l}\text { Base de datos FIC }(B, A) \\
\text { Redes y sistemas } \mathrm{FIC}(\mathrm{B}, \mathrm{A})\end{array}$ \\
\hline $\begin{array}{l}\text { Utilizar ladocumentación y elconocimiento para resolver } \\
\text { desafíosen entornos inf ormacionales. }\end{array}$ & $\begin{array}{l}\text { Base de datos FIC }(B, A) \\
\text { Redes y sistemas FIC }(B, A)\end{array}$ \\
\hline $\begin{array}{l}\text { Aprender a trabajaren grupo colaborando y } \\
\text { respetandoladiversidad y ejerciendolatolerancia y } \\
\text { latransparencia comunicativa }\end{array}$ & $\begin{array}{l}\text { Base de datos FIC }(B, A) \\
\text { Redes y sistemas } F I C(B, A)\end{array}$ \\
\hline 11. Competenciasprofesionales & \\
\hline $\begin{array}{l}\text { Discutir tendencias significativas y desarrollos emergentes } \\
\text { y su impacto social con una actitud crítica y socialmente } \\
\text { involucrada. }\end{array}$ & $\begin{array}{l}\text { Base de datos FIC }(B, A) \\
\text { Redes y sistemas FIC }(B, A)\end{array}$ \\
\hline $\begin{array}{l}\text { Involucrarseenelaprendizaje continuo y lainvestigación, } \\
\text { valorar críticamentelosnuevosescenarios y propuestas. }\end{array}$ & $\begin{array}{l}\text { Base de datos FIC }(B, A) \\
\text { Redes y sistemas } \mathrm{FIC}(\mathrm{B}, \mathrm{A})\end{array}$ \\
\hline $\begin{array}{l}\text { Habilidadendesarrollar soluciones innovadoras a } \\
\text { problemas nuevos o escenariosnuevos. }\end{array}$ & $\begin{array}{l}\text { Base de datos FIC }(B, A) \\
\text { Redes y sistemas } \mathrm{FIC}(\mathrm{B}, \mathrm{A})\end{array}$ \\
\hline 12. Desempeñoprofesional y ético & \\
\hline $\begin{array}{l}\text { Comprenderlasimplicancias éticas de lagestión de } \\
\text { lainformación y lasrepercusionessociales y políticas de } \\
\text { lamisma }\end{array}$ & $\begin{array}{l}\text { Base de datos } \mathrm{FIC}(\mathrm{B}, \mathrm{A}) \\
\text { Redes y sistemas } \mathrm{FIC}(\mathrm{B}, \mathrm{A})\end{array}$ \\
\hline $\begin{array}{l}\text { Tener una conductaprof esional ética e involucrada } \\
\text { socialmente }\end{array}$ & $\begin{array}{l}\text { Base de datos FIC }(B, A) \\
\text { Redes y sistemas } \mathrm{FIC}(\mathrm{B}, \mathrm{A})\end{array}$ \\
\hline $\begin{array}{l}\text { Promover losdesarrollosprof esionales y éticos de las } \\
\text { sociedades profesionalesnacionales e internacionales. } \\
\text { (IFLA e ICA) }\end{array}$ & $\begin{array}{l}\text { Base de datos FIC }(B, A) \\
\text { Redes y sistemas FIC }(B, A)\end{array}$ \\
\hline $\begin{array}{l}\text { 13. Demostrar eficiencia laboral, creatividad y } \\
\text { emprendedurismo }\end{array}$ & \\
\hline $\begin{array}{l}\text { Desarrollarmejoras y propuestasenlos aspectos } \\
\text { informacionalesenlosprocesos y losservicios. }\end{array}$ & $\begin{array}{l}\text { Base de datos FIC }(B, A) \\
\text { Redes y sistemas FIC }(B, A)\end{array}$ \\
\hline $\begin{array}{l}\text { Desarrollarel rol delprofesional de } \\
\text { lainformaciónenelcumplimiento de lamisión y los roles de } \\
\text { laorganizaciónenla que se desempeña }\end{array}$ & $\begin{array}{l}\text { Base de datos FIC }(B, A) \\
\text { Redes y sistemas FIC }(B, A)\end{array}$ \\
\hline
\end{tabular}

